\title{
Téoros
}

Revue de recherche en tourisme

\section{L'évolution des parcs thématiques : de la mécanique au numérique}

\section{Jacques Bernier}

Volume 14, numéro 3, automne 1995

Tourisme et technologies de l'information

URI : https://id.erudit.org/iderudit/1075087ar

DOI : https://doi.org/10.7202/1075087ar

Aller au sommaire du numéro

Éditeur(s)

Université du Québec à Montréal

ISSN

0712-8657 (imprimé)

1923-2705 (numérique)

Découvrir la revue

Citer cet article

Bernier, J. (1995). L'évolution des parcs thématiques : de la mécanique au numérique. Téoros, 14(3), 40-44. https://doi.org/10.7202/1075087ar d'utilisation que vous pouvez consulter en ligne.

https://apropos.erudit.org/fr/usagers/politique-dutilisation/ 


\title{
L'ÉVOLUTION DES PARCS THÉMATIQUES : DE LA MÉCANIQUE AU NUMÉRIQUE
}

\author{
Jacques Bernier
}

\author{
Jacques Bernier \\ est président du Centre \\ Thématique Numérique \\ Canada Inc. \\ (e-mail: bernier@teleport.ca) \\ (http://www.virtualworld.com)
}

\section{Introduction}

La revue Téoros d'automne 1993 portalt sur "Le renouveau des parcs à thèmes", La majorité đes articles traltalent de questlons ayant tralt aux parcs à thèmes de type traditionnel ou mécanlque. Par contre, certalns artıcles soulignalent défà l'émergence d'une nouvelle gếnératlon de parcs à thème de type numérlque rendue posslble grâce aux nouvelles technologles. Dans le présent numéro de Téoros consacré à la technologle, l'occaslon nous est apparue bonne d'approfondir le sujet des parcs thématlques de type numérlque. Mals II y a plus. C'est également l'occasion de saluer l'ouverture au centre-ville de Montréal. en aô̂t 1995, d'une nouvelle attraction touristique le Monde Vrtuelme, laquelle appartlent à cette nouvelle génératlon de parcs à thèmes.

Il est utile de rappeler, d'entrée de jeu, la dénnıltion d'un parc à thèmes ou thématique telle que présentée par Suzanne Chassé (Téoros, automne 1993) :

Un parc à thèmes est un lleu de lolsir consacré à la distraction et aux jeux; un espace clos organlsé autour d'un ou plusleurs thèmes; un site où la vocatlon commerclale est Importante (restauratlon, boutlques, etc.): un lleu conçu pour tous les membres de la famllle: un aménagement requérant des Invesussements majeurs.

Cette définltion s'applique en grande partle au Monde Vituelmo. Mals avant de présenter cette nouvelle attractlon à Montréal, au premler rang au Canada par son envergure, II convlent de défnir brlèvement les principales dlistinctions entre les parcs mécanlques et les parcs numériques, alnsi que de cerner la nouvelle technologle connue sous le nom de Réalité Virtuelle.

\section{Parcs mécaniques vs parcs numériques}

Les parcs thématlques mécanlques requlèrent beaucoup d'espace; les parcs numérlques, molns. Les parcs mécanlques sont habituellement situés en banlleue. Une tendance nette se dessine qul favorise l'implantatlon de petits parcs thématiques en milleu urbaln, notamment les parcs numérlques. Ces dernlers peuvent être Implantés au coeur de la ville. Cette nouvelle génération de parcs à thème s'harmonlse avec le mouvement de revitallsation des grands centres urbains.

Les grands parcs de style Walt Disney ne sont pas prêts d'être reprodults dans un proche avenir. Premlèrement II sont très coûteux à bâtir. Ensulte, lls prennent beaucoup d'espace. Les parcs mécanlques utllisent des atomes: plus ça en prend, plus ça coûte cher. Par contre, les parcs numériques utulisent des bits; plus les bits sont utilisées ou recyclées plus grandes est leur valeur.

Avec les parcs mécanlques, II y a un risque que les goûts du public changent plus rapldement que le temps requis pour récupérer l'Investlssement en Immobllisation. Les manèges mécanlques (montagnes russes ou autres) ne peuvent être modiflés une fols Installés. Les petits parcs numérlques offrent une alternative molns coûteuse, plus flexlble au chapltre de la programmation et plus adaptée à une tendance de fond touchant les nouvelles formes de divertlssement.

Les parcs mécanlques font référence au monde physlque réel, au monde bâtl en trols dimenslons. Tandls que les parcs numérlques se réfèrent à la réallté virtuelle.

\section{Réalité virtuelle}

La réallté virtuelle est une technologle toute récente qui a falt son apparltion aux États-Unls vers le mllleu des années 1980. D'abord mlse au polnt par l'industrle milltalre pour les centres d'entraînement. la réallté virtuelle a peu à peu falt l'objet d'une couverture médlatlque Incroyable. Des revues aussi prestlgleuses que Thme et Fortune, y consacrent de nombreux articles tout en falsant ressortir le grand potentiel commerclal de la réallté virtuelle. II n'en fallalt pas plus pour que les grands du monde du divertissement s'Intéressent à cette techologle et y trouvent des exploitations commerclales.

La réalıté virtuelle est de plus en plus présente dans l'ımaglnalre populalre. Deux facteurs ont contrlbué à cette popularIté: en plus de la couverture médlatlque, mentlonnons les productions clnématographlques où l'on nous montre des gens ordinalres transformés en champlons.

Qualıfiée d'Inventlon gếnlale pour les parcs d'attractlon et les centres de dlvertlssement. la réalltés virtuelle ne relève plus de la sclence-flctlon et s'impose enfin comme un médlum de divertlssement aux possibllités infnnles. Elle fascine parce que, tout comme le cinéma au début du slècle. elle frappe l'Imaginalre des gens en leur permettant de vive des émotlons dans des unlvers fantastlques recréés de toutes plèces.

Avec les Jeux vidéos et les plus récents manèges de simulatlon des parcs d'amusement, la réalitế virtuelle constltue la toute derulère attractlon de l'industrle du "dlvertlssement technologlque". 
Au sens large, la réallté virtuelle permet à un usager d'évoluer dans un unlvers Imaginaire. Généré par ordinateur, cet unlvers intègre des Images 3-D en temps réel et des effets sonores qul donnent au particlpant la sensation qu'll Interagit, d'une façon qui simule la réalité, avec l'environnement alnsi recréé.

Le particlpant vit alors une expérlence où Il peut Interaglr à la fols avec les composantes du jen et dans certalns cas avec d'autres joueurs. Dans ces cas, la dynamlque d'interaction de groupe contrlbue autant, sinon davantage, à l'expérlence que le jeu lul-même.

La réallté vlrtuelle présente un grand potentiel d'attraction pour l'Industrle du divertissement qul se plaît à créer des envlronnements fantastlques contrôlés où les usagers peuvent voyager dans l'Irréel. Les gens alment se falre divertir. Ils veulent de la magle, du fantastlque, de l'évaslon, le tout sans risque. En permettant à I'usager un très haut degré d'Interactivité, la réalité virtuelle contlent plusleurs Ingrédients llés au succès d"une activité divertissante comme par exemple l'immerslon dans un autre monde, la compétition avec d'autres joueurs, la posslbilité de converser en temps réel, le pouvolr de contrôler ses actions et de prendre des déclslons, la possibllité d'améllorer ses compétences et de devenir de plus en plus hablle dans le jeu, etc. Mals surtout, le principal attrait réside dans le falt qu'avec la réalthé virtuelle, le joueur ne regarde pas le héros, Il est le héros.

Les nouvelles attractlons de réallté virtuelle font appel à des équlpements Informatlques hautement sophistlqués qul n'étalent accessibles Jusqu"à récemment qu'à une toute petite minorlté dans les laboratolres de recherche des grandes entreprises aérospatlales ou militalres. Mettant à profit une pulssance peu ordinalre de traltement de données, d'ambitleux concepteurs de jeux sont en train de créer des expérlences interactives pour usagers multiples qui redéfinlssent entlèrement les frontıères entre le Jeu, le manège, le clnéma et le sport de compétition.
Coûteuses à construlre, les attractlons de réallté vlrtuelle sont conçues de façon à être réutillsées avec le même équipement mals avec différents programmes (logiclels) pour créer de nouveaux Jeux et de nouvelles expérlences, tout comme dans une salle de cinéma où l'on présente de noulveaux films en utillsant le même équipement de projectlon.

La réallté virtuelle se présente sous différentes formes. Il y a la technologle utulsant des casques de visuallsation et des gants tactiles (head-mounted display); les cablnes d'Immerslon (pod-based): I'Insertion de partlcipants dans des paysages virtuels au moyen de la télévision (walk-through TV envlronments).

Les prodults de divert.lssement technologlque peuvent être classés dans deux grandes catégorles: passifs et interactifs.

Les prodults de divertissement technologlque "passifs" comprennent les films grand écran à haute résolution qul ont falt leur apparition au début des années ' 80 . tels les fllms Imax et la dernlère génératıon de manèges de simulatlon comme Star Tours aWalt Disney et Back to the Future a Universal Studlos. Ces films ou manèges donnent au visiteur l'impression d'étre au coeur de l'actlon grâce à des effets spéclaux d'Images, de sons et de mouvement. Le résultat est cependant toujours le même : l'auditolre ne peut changer le cours de l'action, et ciest toujours le même effet qul est créé.

Les prodults de divertissement technologique "Interactifs" ne peuvent être générés qu'en utilisant des Images par ordinateur pour reprodulre un environnement en temps réel. Et c'est là qu'intervlennent les aventures de réallté virtuelle (avec ou sans mouvement) où laction des joueurs permet de modifler l'expérlence et de changer le cours de l'histolre. Le réallsme des Images et le son trldimensionnel, combinés à la magle d"une Interactivité totale, sont les trols éléments-clés de ce prodult de divertissement.

Tous convlennent que la réalité virtuelle sera un des éléments qui feront partie de notre quotidien au cours du 


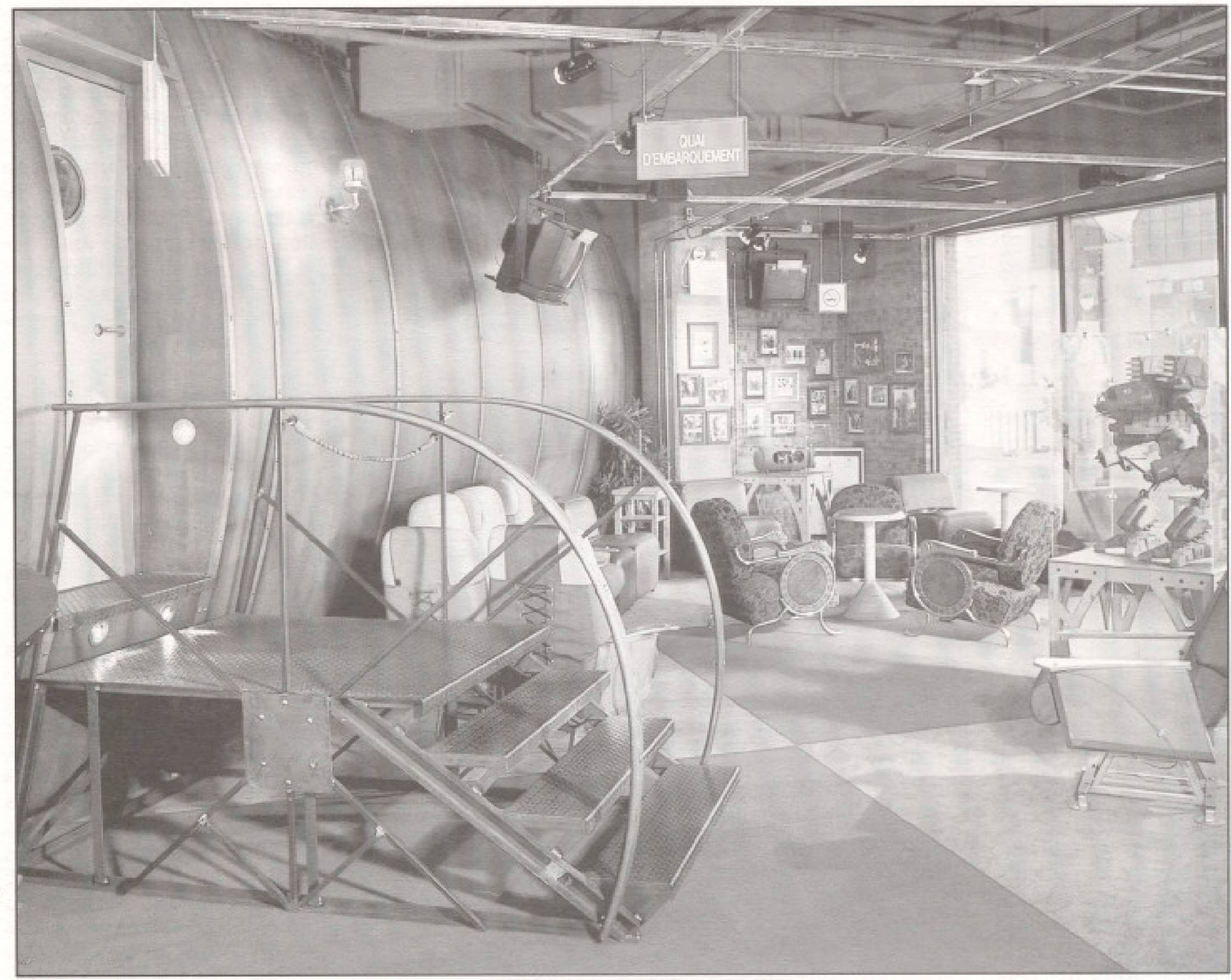

prochaln millénalre, que ce solt au nlveau médical, ěducalif. des affalres et du divertissement. Pour s'en convalncre, II s'aglt de se référer à un certain nombre de tendances de fonds que sont, par exemple dans le domalne du dlvertlssement, la numértsation de la réalité, la progression d'appropriation par la population des nouvelles technologles. I'Interactlvité et le besoln d'actlvités exaltantes (adrénaline) mals sans rlsque.

La réalité virtuelle est encore au stage de l'enfance tant du polnt de vue technologique que de son utilisation comme divertissement dans les parcs à thèmes. Louls M. Brlll (Funworld, Julllet 1992) a recensé un certain nombre de caractéristiques et de problèmes llés aux diverussements de réalité virtuelle que nous résumons alnsl :

\section{Les principales caractéristiques}

- tout en consommant peu d'espace pliysique, un environnement viruel peut crér électronlquement des paysages représentant des centaines de kllomètres de territolre;

- le public peut jouer à plusleurs reprises dans un environnement virtuel sans nécessalrement vive lá mêmé expérlence;
- le degré de difflculté peut être modiné facllement et rapldement;

- le changement des aventures ou des expérlences ne nécessite pas une nouvelle construction, mals tout simplement de charger différents logiclels;

- les espaces virtuels n'impliquent pas l'utilisation d'une langue et sont intultifs, ce qul en falt des prodults accessibles a des cllentèles tourlstiques de différentes langues et de differentes cultures.

Certains équipements ou environnements de réalité virtuelle, dans leur conception inltiale, ne sont pas exempts de problèmes. Parm I les problèmes rencontrés, mentionnons

- certains équipements peuvent présenter des problèmes d'liyglène étant donné qu'lls sont partagés par un grand nombre de personnes par jour;

- certains équipements manquent de sophlstication, et par conséquent ne représentent pas un grand attrait;

- les joueurs trouvent souvent que leurs réactions en temps réel ne: recolvent. pas de réponses suffisamment rapldes de la part de leur partenalre virtuel; 
- certains équipements, par exemple des casques, posent des problèmes à cause de leur polds on de leur talle. sans compter les délals pour ajuster l'équlpement à chaque personne:

- la difficulté d'utulisation des "joysticks" comme instıument de navlgation ou comme arme varle selon le type de jeu ou selon l'expérlence du joueur.

\section{Enjeux financiers importants}

La revue Business Week du 14 mars 1994 quallflalt l'industrle du divertissement comme l'industrle de crolssance des années "90. Larticle avait pour titre: "The entertalnment economy, America's growth engines : theme parks, casinos, sports, Interactive TV.

En 1994. les Américains ont dépensé 340 milliards de dollars en divertissement et activités récré́atives.

A titre Indicatif, volol l'importance relative de différentes composantes de l'industrle américalne du divertissement (en millards de dollars U.S.) selon les estumés publiés dans les magazines Thme, Wred et Fortune :

Cinéma

Sports (spectateurs)

Parcs d'amusement

Jeux vidéos

Arcades électronlques

Locatton de vildéos

Câble

Jeux d'argent (gambling)

Llvres, magazines et journaux

A noter que le marché des jeux vidéos dépasse en volume celul du clnéma. On prévolti qu'll attelndra 7.5 mllliards \$ U.S. en 1996. Cette crolssance phénoménale attire les supers corporatlons comme AT\&T, Matsushita, Paramount. Vlacom, Blockbuster, IBM, Silicon Graphics et Timer Warner quil tentent acturellement de pénétrer cette Industrie dominée |usqu'lol par les deux géants laponals Nintendo et Segn.

\section{Monde virtuelmic}

\section{Le produit}

Le Monde Virtuelme est aménagé à l'Intérleur du Complexe Desjardins au centre-ville de Montréal. Les vislteurs peuvent y accéder solt par la rue Salnte-Catherlne (en face de la Place des arts) ou par I'intérleur du Complexe. Le Monde Vrrtuelmo est un produit se situant à la convergence des télécommunlcatlons, de l'audlo-vlsuel, de l'Informatloue et du divertissement. Sa réalisalion a requis un investissement de trols millions de dollars.

Les aventures sont intégrées dans un environnement. thématique global qui comprend un resto-bar sans fumée nl alcool. où les membres-cllents peuvent échanger sur laventure et leur performance, une boutlque où l'on vend des prodults rellés aux scénarlos des aventures, un décor bizarre et mythlque qul mélange deux cultures flctlves, celle des premlers grands scientistes et explorateurs, et celle des héros des galaxles de l'an 3000. En entrant dans l'aventure. les membres-cllents font la découverte de la Ligue géographlque virtuelle, une soclếté secrète fictive fondée en 1897 par Alexander Graham Bell pour explorer d'autres univers et quil regroupalt les plus grands cerveaux de notre slècle tels EInstein et Nikola Tesla ou de grands aventurlers tels Charles Lindbergh et Howard Hughes. La Ligue fêtera son centenalre en 1997.

C'est comme au théfitre, ou presque, II y a une histolre, un décor, des éclalrages, une mlse en scène, des acteurs (les participants eux-mêmes): on peut alnsı parler de thétre Interactif. Le spectacle se déroule en quatre actes. Acte 1: vlsionnement d'un court fllm de divertlssement. Acte 2: préparatıfs a la mlssion: des guldes-techniclens vous expllquent l'aventure et dans quel unlvers vous allez vous retrouver. Acte 3: le déroulement de la "mission" à l'intérteur d'une cabine spéclale. Acte 4: session de debriefing au cours de laquelle les participants passent en revue les péripétles de l'actlon. Cet ensemble crée une amblance d'aventure et favorlse une dynamlque soclale facllitant la communlcation entre les lummains.

\section{Apercu des programmes actuels et futurs}

Red Planetmc, la premlère aventure disponible au Monde VIrtuelmc, vous falt particlper à une course dans les corrldors minlers de la Planète Mars en l'an 2053, aux commandes d'un aéroglsseur surpulssant. Pour plmenter un peu les choses. les amateurs ont mls au polnt une varlante, le football martlen, quil s'apparente au "Roller Derby".

La seconde aventure. du nom de Battletech8, place le pllote à l'intérieur d'un robot lumanoïde haut de 30 pleds appelé Mech. qui pourchasse et combat ses congénères sur la planète déserte Solarls VII.

Le Monde VIrtuelme contlnuera de se renouveler et de s"améllorer. Alnsı. au début 1996, on introdulra une nouvelle aventure laquelle entrainera les particlpants à la découverte d'Atlantls.

\section{La clientèle}

La programinat Ion actuelle du Monde Virtuelmo attire malorltalrement des jeunes de $18-34$ ans Intéressés par des aventures se déroulant daus un cadre à la fols exaltant et sécurltalre. Ces jeunes sont passlonnés par des expériences oì l'on retronve à la fols l'esprit communautalre et l'esprit sportif. A une eporue où le monde est accessible de notre salon. Ils ont le désir de partager des expérlences plus que famals. Avec l'ajout éventuel de nouveaux programmes, le Monde VIrtıelme vise, à terme, à reloindre également la famlle avec de jeunes enfants.

Au chapitre de l'achalandage. le Monde Vrtue lma s'attend à aturer entre 70 000 à 80000 personnes dès la premlère année d'opérat.lon. Comme claque personne effectue en moyenne trols mlsslons sur une base anmuelle. on prévolt quelque 235000 mlsslons ou transactions an cours des premlers douze mols.

\section{Les impacts}

Trente-cing emplols - certalns à temps plein. d'autres à temps partiels, sont créés par le Monde Virtuelme. De ce nombre. chn sont des postes cadres et trente, des postes reliés à l'opération. 
Le Monde Virtuelme constitue un divertissement permettant d'éduquer les public montréalals et les visiteurs à la réallté virtuelle. Centre Thématlque Numérique Canada(1), proprlétalre de la marque de commerce Monde Virtuel, a importé la plus récente technologle amérıcaine détenue par Vırtual World Entertainment ${ }^{\mathrm{TD}}$, technologle Implantée pour la premlère fols dans le monde à Montréal. Avec le temps, on peut
Finalement, nous avons discuté du prodult, de la clientèle et des Impacts du Monde Virtuelmc. Les Impacts mentlonnés ont trait a l'éducation populalre en regard des nouvelles technologles, à la création d'emplols et aux nouvelles opportunités d'affalres, tant sur le marché domestıque que sur les marchés extérleurs, pour nos créateurs de loglclels et nos designers d'aménagement intérieur.

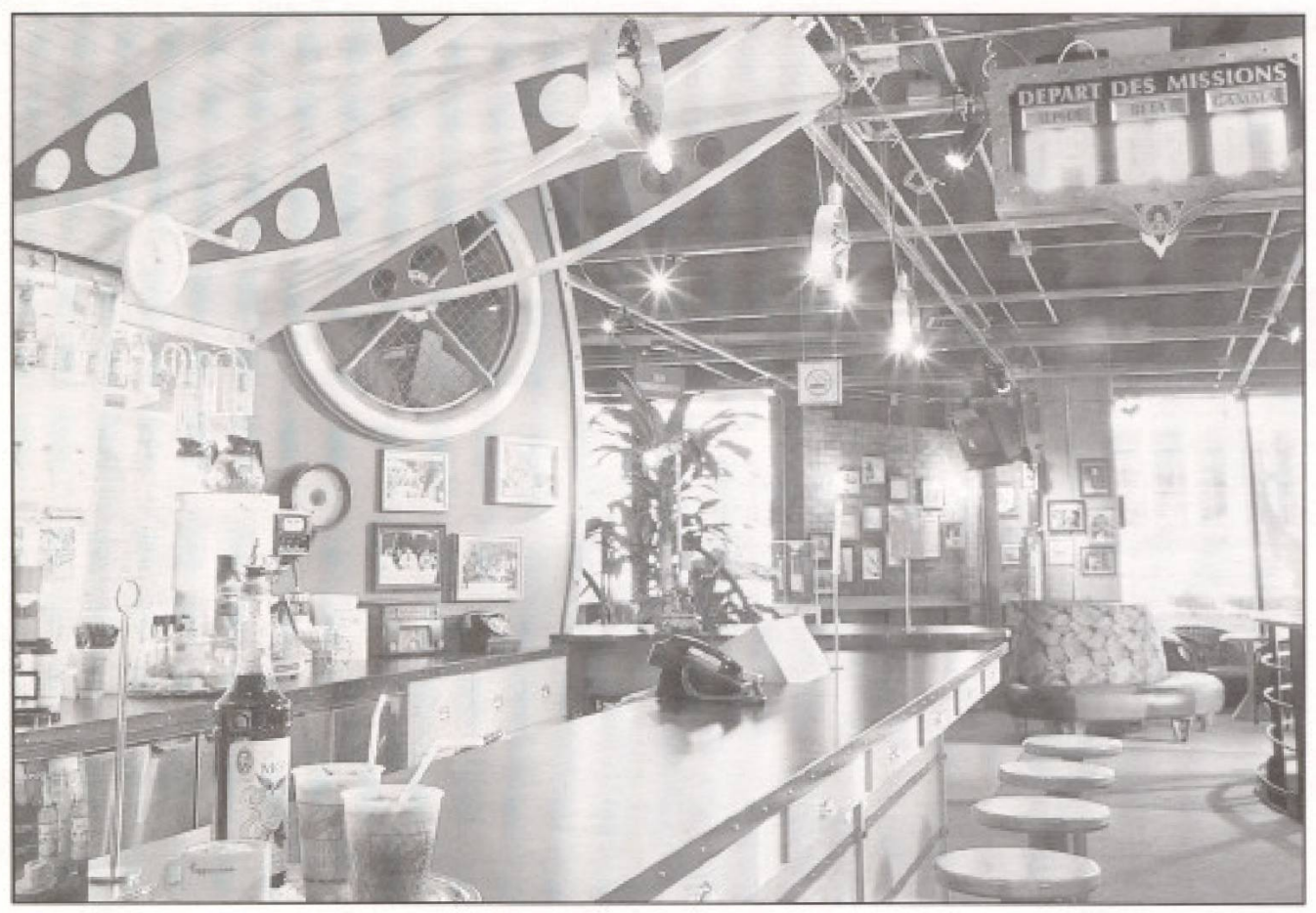

s'attendre à ce que des entreprises montréalalses s'lntéressent à développer des logiclels proposant de nouvelles expérlences de réalité virtuelle; à noter que ces loglclels seralent destinés tant pour le réseau national que pour le réseau International du Monde Virtuelmc / Virtual WorldTo.

Constatant à la visite des centres américalns de Virtual World Entertainment ${ }^{\mathrm{TD}}$ que l'aménagement intérleur de ces centres n'étalt pas adapté à la culture montréalalse, les dirlgeants du Monde Virtuelmc ont conflé à des créateursdeslgners de Montréal - Fotl \& Drou in Designers, le mandat de concevolr et réallser un nouvel aménagement Intérleur tout en respectant le scénarlo mls au polnt par Virtual World Entertalnment ${ }^{\mathrm{TD}}$. Le travall exécuté peut être considéré de succès. On parle même d'exporter dans d'autres centres du réseau mondial de Virtual World EntertalımentTo certalns objets créés par cette flrme de designers montréalals.

\section{Conclusion}

Dans cet article nous avons tout d'abord rappelé la déflnItıon des parcs à thèmes telle que présentée dans l'éditlon de l'automne 1993 de Téoros. Par la sulte, nous avons falt ressortır brlèvement les principales distinctions entre les parcs thématıques traditlonnels de type mécanlque et les nouveaux parcs de type numérique dont l'essor' est principalement dî à la technologle de la Réalité Virtuelle. Nous avons décrit celte technologle utilisée par le Monde Virtuelmo, nouvelle attraction touristique Inaugurée àMontréal en aont 1995.

\section{Références}

BILL, M. Brill, Welcome to the VIrtual Real World, Funworld, IAAPA, julllet 1992

Buslness Week. The entertainment economy. America's growth englnes: Theme parks, casinos, sports, interacUve TV, 14 mars 1994

CHASSÉ, Suzanne, Les parcs thématıques et Je tourtsme, Téoros, vol. 12, 3, octobre 1993

CRAVENS, Tood, Playing game with technology. Funworld. IAAPA, mal 1995

FORTUNE. 27 dlécembre 1993

GRAFF, Joln. Amusement on the Superhigway, Park World. The World's Fair Ltd, février 1995

TIME, 27 septembre 1993 et 14 mars 1994

WIRED, décembre 1993

Note

(1) Centre Thématique Numérique Canada inc. a obtenu les droits canadlens nour les maryues de commerce, les lapiciels et. les technologies de Virtual World Entertainment ${ }^{\mathrm{TD}}$. Le Monde Virtuelme est une marque de conmeroe utilisée par Centre Thématique Numérique Cansda. 